Submitted to Ap.J.

\title{
NGC 4138 - A Case Study in Counterrotating Disk Formation
}

\author{
Aniruddha R. Thakar, Barbara S. Ryden ${ }^{1}$ \\ Department of Astronomy, The Ohio State University, Columbus, OH 43210-1106 \\ email: thakar.4@osu.edu; ryden@payne.mps.ohio-state.edu \\ Katherine P. Jore, Adrick H. Broeils ${ }^{2}$ \\ Center for Radiophysics and Space Research, and National Astronomy and Ionosphere Center ${ }^{3}$, \\ Cornell University, Ithaca, NY 14853 \\ email: jore@astrosun.tn.cornell.edu, broeils@astro.su.se
}

\begin{abstract}
The Sa(r) galaxy NGC 4138 has been recently found to contain an extensive counterrotating disk which appears to be still forming. Up to a third of the stars in the disk system may be on retrograde orbits. A counterrotating ring of H II regions, along with extended counterrotating H I gas, suggests that the retrograde material has been recently acquired in the gas phase and is still trickling in. Using numerical simulations, we have attempted to model the process by which the counterrotating mass has been accreted by this galaxy. We investigate two possibilities: continuous retrograde infall of gas, and a retrograde merger with a gas-rich dwarf galaxy. Both processes are successful in producing a counterrotating disk of the observed mass and dimensions without heating up the primary significantly. Contrary to our experience with a fiducial cold, thin primary disk, the gas-rich merger works well for the massive, compact primary disk of NGC 4138 even though the mass of the dwarf galaxy is a significant fraction of the mass of the primary disk. Although we have restricted ourselves mainly to coplanar infall and mergers, we report on one inclined infall simulation as well. We also explore the possibility that the $\mathrm{H} \alpha$ ring seen in the inner half of the disk is a consequence of counterrotating gas clouds colliding with corotating gas already present in the disk and forming stars in the process.
\end{abstract}

Subject headings: galaxies: spiral — galaxies: structure — galaxies: evolution galaxies: interactions — galaxies: kinematics and dynamics — hydrodynamics

\footnotetext{
${ }^{1}$ National Science Foundation Young Investigator.

${ }^{2}$ Present address: Stockholm Observatory, S-13336 Saltsjöbaden, Sweden

${ }^{3}$ The National Astronomy and Ionosphere Center is operated by Cornell University under a cooperative agreement with the NSF.
} 


\section{Introduction}

NGC 4138, an early-type spiral galaxy within the Virgo supercluster, has no distinguishing feature other than a nuclear ring of star-forming H II regions similar to rings seen in many other early-type spirals (see, for example, Pogge \& Eskridge 1993). Nevertheless, it has recently joined the growing list of spiral galaxies that are now known to possess two disks which rotate opposite to each other. The spiral/S0 galaxies currently known to have such gaseous and/or stellar counterrotating disks are NGC 4550 (Rubin, Graham \& Kenney 1992), NGC 4826 (Braun, Walterbos \& Kennicutt 1992), NGC 7217 (Merrifield \& Kuijken 1994), NGC 7332 (Fisher, Illingworth \& Franx 1994), NGC 4546 (Galletta 1987), NGC 3626 (Ciri, Bettoni \& Galletta 1995), NGC 3593 (Bertola et al. 1996) and most recently, NGC 4138 (Jore, Broeils \& Haynes 1996, hereafter JBH). This latest instance of a counterrotating disk was discovered as part of a survey of "boring" spiral (Sa) galaxies which had no unusual morphological features. The counterrotating disk consists of both stars and gas which add up to about a third of the mass of the primary disk, and it appears to be still forming or has formed recently, as evinced by the extended counterrotating neutral gas surrounding the primary disk and the nuclear ring of star-forming H II regions which is also counterrotating (assuming that the counterrotating material does not remain in the gas phase for long). An $\mathrm{H} \alpha$ emission-line imaging survey of S0 galaxies Pogge \& Eskridge 1987) previously detected the inner ring of H II regions in NGC 4138, which is classified as $\mathrm{S}^{+}$ by the Third Reference Catalogue of Bright Galaxies (de Vaucouleurs et al. 1991) although the Revised Shapley-Ames Catalogue (Sandage \& Tammann 1981) classifies it as Sa(r). A follow-up study (Pogge \& Eskridge 1993) confirmed the existence of the ring, resolving many "blobs" into discrete H II regions.

The examples of counterrotating disks currently known run the gamut from counterstreaming stars (e.g., NGC 4550) to counterstreaming gas (e.g., NGC 7332), and this variety among the specimens, along with the observed differences in the velocity dispersions of the two components, strongly implies an external (merger or slow accretion) origin for the retrograde material. The presence of ionized gas along with stellar counterrotation suggests that at least some of this material was in the gas phase when accreted and formed stars later. However, in the cases where the retrograde disk mass is comparable to the primary prograde disk mass, the merger or accretion theory invoked must explain how the primary disk can remain unperturbed in spite of the influx of a large amount of stars and gas. Simulations of major mergers show clearly that the acquisition of a large number of stars is quite a disruptive process which leaves a remnant that resembles an elliptical galaxy more than a disk galaxy (e.g. Barnes 1992; Barnes \& Hernquist 1992a). This is true even for gas-rich mergers between disk galaxies that yield a counterrotating disk (Hernquist \& Barnes 1991). We can therefore rule out major mergers in which a large amount of dissipationless matter is dumped into the galaxy within a relatively short time. This leaves minor gas-rich mergers and secular gas infall as the processes most likely to produce massive counterrotating disks without heating up the primary disk inordinately.

Whereas examples of counterrotating gas in S0s and spirals have been known for some time (see Sage \& Galletta 1994 and references therein), the unexpected discoveries of a few massive, stellar counterrotating disks in galaxies previously thought to be "normal" naturally raise the troubling question: how many more have been missed? Systematic searches for counterrotation in 
spiral galaxies are just getting under way, and so far the results are mixed. A recent survey of 28 S0's with high signal-to-noise spectra (Kuijken, Fisher \& Merrifield 1996) revealed no new cases of stellar counterrotation but uncovered counterrotating gas in almost a quarter of the sample. This implies that most if not all counterrotating disks must start out as gas disks, and suggests that star formation may somehow be inhibited in the retrograde gas. Furthermore, most of the counterrotating gas disks are substantially smaller than the corresponding primary disks. These findings argue against a high frequency of massive, stellar counterrotating disks in S0 galaxies. Theoretical studies of the origin of massive counterrotating disks are necessary to complement the observations and make some predictions which can be checked against future observations.

We have been investigating the origin of such massive counterrotating disks in spiral galaxies with numerical simulations in which we try to produce a massive counterrotating disk in a fiducial spiral galaxy (Thakar \& Ryden 1996, hereafter TR). However, given the large number of input parameters that can have an appreciable effect on the outcome, it is of great practical value to adopt a phenomenological approach and study individual galaxies so that we can narrow down the range of parameters that are relevant to existing counterrotating disks.

As in TR, we investigate two mechanisms here for producing a counterrotating disk in NGC 4138. The first process is gradual or secular gas infall, and the second is a gas-rich dwarf satellite merger. Minor mergers and accretion have been receiving increased attention in the last few years, due partly to the fact that major mergers have been shown, through numerical simulations, to be unable to produce some of the observed features in spiral galaxies, and certainly can be ruled out in cases where cold, thin disks still dominate the galaxy morphology. The picture that is emerging is that the importance of accretion and minor mergers as a means for spiral galaxy evolution may have been underestimated. The secular evolution of galaxies most likely is driven by such events from later types towards S0 (e.g. Walker, Mihos \& Hernquist 1996). There is also some evidence from numerical experiments to support the idea that spiral disks are more resistant to minor mergers if the angular momentum of the merger orbit is high enough for the primary disk to respond in a coherent manner by tilting rather than warping or heating (Huang \& Carlberg 1996).

The prevalence of counterrotating gas disks is clearly indicative of the importance of gas in the process(es) responsible for forming them. Indeed, TR found it quite impossible to produce a counterrotating stellar disk within a Hubble time from a dissipationless merger without wrecking the primary disk. However, it is not clear whether it is necessary for all the retrograde matter accreted to be in the form of gas. A gas-rich dwarf merger may work nearly as well as pure gas infall in some cases, especially if the dwarf has a low M/L ratio. There are some difficulties associated with secular gas infall, particularly the source of the infalling gas and the need for the gas to maintain retrograde angular momentum with respect to the primary disk over a good fraction of a Hubble time. Alternatives to gas infall, of which gas-rich dwarf mergers is the most promising, must be thoroughly investigated to determine their ability to produce massive counterrotating disks.

Based on numerical experiments with a cold and thin primary, TR found that gas infall was able to produce massive counterrotating disks without excessive heating of the primary disk, but the same could not be said of gas-rich dwarf mergers, which were found to be problematic. For 
the significantly hotter and more compact primary of NGC 4138, gas-rich dwarf mergers do a much better job, and in fact produce a counterrotating disk in a significantly shorter time than continuous infall. We briefly describe our numerical method in section 2. Our simulation results are presented in section 3. Analysis of these results and comparison with previous work in galaxy interactions are in section 4 . Section 5 lists our conclusions.

\section{Computational Method}

The numerical method used is described in detail in TR. We use a tree code to compute the gravitational forces for all particles and a sticky particle dissipation scheme for the gas dynamics. We started with a basic version of the tree gravity solver (Barnes \& Hut 1986) written in the C language and optimized it for execution on the Cray Y-MP at the Ohio Supercomputer Center. To this we added a sticky particle code which implements a simple form of gas dynamics via inelastic collisions which dissipate kinetic energy and conserve linear and angular momentum. Our sticky particle code is a combination of previous schemes (Brahic 1977; Schwarz 1981; Negroponte \& White 1983), and is also optimized for the Cray Y-MP.

For the simulations described here, we use twice the number of particles to represent the halo (32k instead of $16 \mathrm{k}$ as in TR). The softening length is smaller $(0.5 \mathrm{kpc}$ instead of $1 \mathrm{kpc}$ used by TR) to give a better resolution. The primary stellar disk is represented as before with $32 \mathrm{k}$ particles. It is dissipationless initially and contains no gas (except for infall simulation I6, see below). The number of gas particles is also the same, being $20 \mathrm{k}$ for gas infall models and 15k for dwarf merger models. The dissipationless matter in the dwarf galaxy is represented by a varying number of particles depending on its mass. The mass of each dissipationless particle in the dwarf is comparable to the mass of each particle in the primary disk $\left(\sim 6 \times 10^{5} M_{\odot}\right)$. The total number of particles is $84 \mathrm{k}$ for the infall simulations and $86 \mathrm{k}$ for the dwarf mergers (except one large $N$ dwarf merger which contains $162 \mathrm{k}$ particles).

Although the two-component model for the spiral galaxy is the same as before, the model parameters are different since we are modelling NGC 4138 instead of a fiducial spiral galaxy. The parameter values for our model of NGC 4138, based on the observations and mass modelling

described by JBH, are summarized in Table 1. The disk in this case is radially much hotter than the disk used by TR, and the value of $Q_{\mathrm{o}}$ (the value of the stability parameter $Q$ at $R_{\circ}=8.5 \mathrm{kpc}$ ) we use is 4.0. This was necessary for the model rotation curve and primary disk velocity dispersion values to be consistent with the "observed" rotation curve and velocity dispersions of NGC 4138 obtained by JBH (Fig. 1). Although the initial galaxy prior to the accretion of the counterrotating material was in all probability different from the one we see today, we had no choice but to start with a model as closely resembling the observed post-accretion galaxy as possible. To investigate what happens if we start with a radially cold primary instead, we ran one dwarf merger simulation with a $Q_{\mathrm{o}}=1.5$ primary disk.

The models for continuous infall and the gas-rich dwarf merger are basically the same as those used by TR. For gas infall, a long (length $=150 \mathrm{kpc}$, width $=10 \mathrm{kpc}$ ), rectangular slab of gas is allowed to fall into the primary spiral galaxy on a retrograde orbit. We chose this model because it allows us to impart the appropriate angular momentum to the gas in a simple manner, 
and because we do not really know what the characteristics of real gas infall are. Our long column of gas (which breaks up into clumps before it falls into the primary galaxy) can be thought of as tidal debris left over from a previous interaction.

The initial conditions and some physical parameters of the infalling gas are different in the present simulations from those used by TR. The velocity dispersion of the gas is higher than before $(\sim 30 \mathrm{~km} / \mathrm{s}$ instead of $\sim 10 \mathrm{~km} / \mathrm{s}$ used in TR), the mass and initial angular momentum of the infalling gas are different to suit the current problem, and we have experimented with the "stickiness" of the gas to assess its impact on the final outcome. We have also studied the effect of an inclined infall orbit and the presence of a small amount of preexisting prograde gas in the disk. The parameter values for the different infall simulations (dubbed I1-I6) are listed in Table 2. The magnitude of the initial angular momentum, $J_{z}$, of the gas can be altered by changing the initial velocity of the gas $V_{0}$ or the initial distance $R_{0}$ of the gas slab from the center of the primary disk, or both. The total mass of the gas falling in is taken to be $50 \%$ of the mass of the primary disk, even though the counterrotating mass observed is at most $30 \%$ of the mass of the disk. The extra gas is included to allow for some gas which is dispersed over large distances and does not fall into the primary disk by the end of the simulation.

Similarly, for the dwarf mergers, the total amount of luminous mass (gas+stars) in the dwarf galaxy is chosen to be $50 \%$ of the primary disk's mass. The dwarf galaxies we have chosen have higher gas contents than do typical dwarfs. Our values for $M_{g} / M_{T}$, the ratio of the gas mass to the total mass, range between 0.27 and 0.53 , whereas the typical range for $M_{H I} / M_{T}$, the ratio of neutral gas mass to total mass, is $0.09-0.24$ for dwarf galaxies (Roberts \& Haynes 1994). However, the latter does not take into account the molecular gas content in dwarf galaxies, which is not very well quantified so far. The mass model for the dwarf galaxy is a spherical Plummer model with the density profile being a polytrope of index 5 (as described in TR). The parameters for the dwarf galaxy in each of the dwarf merger simulations (M1-M3) are listed in Table 3. The total mass of the dwarf galaxy therefore ranges between $\sim 9 \%$ and $\sim 18 \%$ of the mass of the primary galaxy. Although the physical parameters for the dwarf galaxy are identical for M2 and M3, the latter uses a radially colder primary disk and a larger number of particles in the simulation (64k each for the disk and halo, and $34 \mathrm{k}$ for the dwarf galaxy) to minimize numerical heating even further.

In all the simulations presented here, the primary disk is held frozen for $t=0-0.2 \mathrm{Gyr}$, which amounts to $\sim 5$ dynamical times. This is to allow the halo to virialize. The disk and halo are then evolved in isolation for $t=0.2-0.5$ Gyr so that they may achieve equilibrium. The gas column (for infall) or the dwarf galaxy is introduced at $t=0.5$ Gyr. In most cases, the simulation results are shown starting at $t=0.9 \mathrm{Gyr}$, so that the simulation is already in progress. The initial time-step is not shown.

With the simple gas dynamics scheme we are using, we do not attempt to study the internal structure of the gas disks formed in our simulations, and hence we follow each simulation only up to the point where a flat disk-like structure with retrograde orbits is formed. The sticky particle scheme does not give an accurate description of the detailed gas dynamics, so we restrict ourselves to studying the overall characteristics of the objects such as the size and shape of the counterrotating disk formed (if any) and the impact of the accretion or merger on the primary disk. 
For the same reason, we also do not study the effect of including large quantities of preexisting gas in the primary disk with this method. We hope to report on these aspects in the near future using SPH simulations which will provide a more realistic description of the gas dynamics with the inclusion of shocks, viscosity, and eventually even star formation.

\section{Results}

\subsection{Continuous Infall}

Figs. 249 depict the behavior of the infalling gas for the different infall simulations (I1-I6). I1 and I2 compare large initial angular momentum $\left(J_{z}\right)$ infall with small initial angular momentum infall respectively for a moderately dissipative gas (coefficient of restitution $\alpha=-0.5$ ). I3 shows the effect of very low initial gas angular momentum on the production of a counterrotating disk. I4 illustrates infall along an orbit which is considerably tilted with respect to the plane of the primary disk. I5 is the same as I1 but with a different parameterization of the sticky gas which produces a smaller counterrotating disk. Finally, in I6 we show the effect of placing a prograde ring of gas in the disk in addition to the infalling gas. The mass and initial density of the infalling gas are the same in all simulations reported here.

For I1, the large $J_{z}$ case (Fig. 2), the resulting counterrotating disk is more than twice as large as the primary disk. The effect of lowering the initial angular momentum of the gas can be seen in I2 (Fig. 3). This approaches the minimum retrograde $J_{z}$ that the gas can have and still yield a counterrotating disk. Although the counterrotating disk formed is slightly smaller in I2 than in I1, it is still significantly larger than the primary disk.

If $J_{z}$ for the gas is reduced further, a counterrotating disk does not form. (Fig. đ). The evolution of the gas is now very different from the previous two simulations. A shell forms at $t=1.7 \mathrm{Gyr}$, and thereafter the gas falls rapidly back into the primary disk. The infall proceeds at a significantly faster rate, and the side view shows that the end result at $t=3.7$ Gyr resembles a spheroid rather than a disk (only the $x-z$ plane view is shown, but the $y-z$ plane view is almost identical). Fig. 5 provides a comparison of the velocity fields of the primary disk and the gas for the I2 and I3. The velocity field of the gas is not ordered for I3, and it is hard to discern a well-defined orbital direction. The difference also shows up in the comparison of the specific angular momenta of the primary and secondary disks for the two simulations (Fig. 6). Even though the net angular is retrograde for I3, we are clearly getting out of the regime which yields a counterrotating disk.

To investigate the impact of a non-coplanar or inclined infall orbit, we performed one simulation in which the infall proceeds along an orbital inclination of $\sim 30^{\circ}$ with respect to the plane of the primary disk $(x-y$ plane). The results are shown in Fig. : 1 . A few shells can be seen forming early on in the gas, and the later panels show that although a counterrotating disk does appear to form, it is not aligned with the primary disk even by $t=5.7$ Gyr. This is evident from the side views, in which the gas counterrotating disk and the primary disk appear to be inclined by almost $45^{\circ}$ with respect to each other. Furthermore, the primary disk flips by almost $90^{\circ}$ not once but twice, each flip taking $\sim 1.5$ Gyr to accomplish. It is clear that a near-coplanar orbit 
is necessary for the counterrotating gas to settle quickly into the plane of the primary disk. TR experimented with a small orbital inclination $\left(5^{\circ}\right)$ and found it to have no appreciable impact on the outcome.

Although simulations I1-I4 illustrate the general aspects of infall simulations adequately, they use a sticky particle formulation in which the dissipative characteristics of the gas are less than ideal. The resulting counterrotating disk is considerably larger than the primary disk regardless of the initial angular momentum. The radial distribution of the particles also shows a clear underdensity of particles at the inner radii (Fig. 14). Since we observed this behavior even when the gas was made very "sticky" (by choosing a smaller coefficient of restitution $\alpha$ ), we previously concluded that this was a limitation of our infall model (Thakar 1996) rather than an artifact of the sticky particle model chosen for the gas. However, upon choosing a smaller maximum radius for selecting collisional neighbors (instead of a smaller $\alpha$ ), the resulting counterrotating disks were appreciably smaller than before. This parameter change has effectively made the gas more dissipative by strongly favoring collisions between nearby particles (which on the average have higher relative velocities due to their mutual gravity) over those between remote particles, even though the total number of collisions is reduced because of the smaller number of nearest neighbors found for each gas particle. Since preferentially selecting collisions between nearby particles is physically more realistic, we adopt this parametrization for all the remaining simulations discussed below.

The results for the new sticky particle formulation are shown in Fig. 8 for I5, for which all the other inputs are the same as I1. A comparison of the primary disk shown in the first panel of the side view in Fig. 8 with the counterrotating disk at $t=4.1$ Gyr indicates that the two are approximately the same size, with the counterrotating disk being slightly smaller. The enlarged views for the last time-step reveal some of the structure of the disk. The gas is distributed fairly evenly with radius, and most of it is within the radius of the primary disk. The counterrotating disk clearly does not have an exponential profile, and it is tilted by nearly $45^{\circ}$ to the original plane of the primary disk. The primary disk also tilts by a comparable amount, as can be seen in the side views of the primary disk. It is not clear what causes the primary disk to tilt so much, especially when it does not tilt nearly as much when prograde gas is included in it (see below).

The nuclear ring of H II regions is a prominent feature of NGC 4138, but the discovery that it is also counterrotating was a surprise. Although nuclear star formation rings in early type spirals are not unusual, this is the first counterrotating star forming ring known. Could this ring be a result of collisions between corotating and counterrotating gas clouds? If so, why do the resulting star forming regions end up counterrotating? We placed a prograde ring of gas, with one-tenth the mass of the infalling gas, at the same location as the observed ring and ran the infall simulation I6 with everything else being the same as for I5 above. The results are shown in Fig. 9. The apparent thickness of the counterrotating disk in the side view is due to its projected inclination. The only discernible differences between the gas disk formed in this case and the one formed in the previous simulation (without prograde gas) are the size and the inclination of the counterrotating disk with respect to the original plane of the primary. Without prograde gas, the disk is a bit larger and tilts by a considerable amount, whereas with prograde gas, the disk is smaller and suffers much less tilting. It is reasonable for the prograde gas to rob the counterrotating gas of kinetic energy and thereby produce a smaller counterrotating disk, but what could the prograde gas have to do 
with the tilting? We are not quite sure, but some arguments on this subject are presented in $\S 4.1$.

\subsection{Gas-Rich Dwarf Merger}

The dwarf merger model we have used is described in detail in TR. We present the results of three gas-rich dwarf mergers here, the first with very little dark matter (M1) and the remaining two with a moderate amount of dark matter (M2 and M3). All mergers are coplanar and the dwarf galaxy models are identical except for the amount of dark matter. M3 uses the same dwarf galaxy model as M2, but has a colder primary disk and twice the number of collisionless particles as M2..

In the first dwarf merger (M1), shown in Fig. 10, we see a counterrotating disk forming by $t \sim 3.1$ Gyr. Although particles still appear to be falling in at $t=4.5 \mathrm{Gyr}$, these are mostly stellar particles from the dwarf, and almost all of the gas particles have fallen into retrograde orbits around the primary disk by this time. The formation of a tidal bridge and two tidal tails is evident from the top view in Fig. 10. The primary disk tilts in response to the merger, and the counterrotating disk which forms is consequently also tilted with respect to the original plane of the encounter. The sizes of the primary and counterrotating disks are comparable, although there are a few gas particles at larger radii.

With the additional dark matter in the second dwarf merger (M2), the counterrotating disk is produced much faster (Fig. 11). There is less tidal stripping of the dwarf, although as in M1, two distinct tidal tails are formed. By $t \sim 2.5 \mathrm{Gyr}$, most of the gas is within the radius of the primary disk, but it has not quite made it to the plane of the disk yet. The gas disk becomes steadily smaller after this, and a comparison of the panels for the last time-step in M1 and M2 shows that the gas disk in M2 is more compact and less inclined to the original plane of the primary. However, even by $t=4.5 \mathrm{Gyr}$, the counterrotating gas does not appear to be very flat, and its apparent thickness is not due to a projected inclination.

The two dwarf mergers are compared in Fig. 12. Although the counterrotating disk is tilted in M1, it is not as thick as it appears because it also tilted in the $y-z$ plane. Such is not the case for the counterrotating disk in M2, which actually is thick. The second merger produces a puffier counterrotating disk whose velocity field is not completely circularized. A comparison of the angular momenta of the two disks shows this more clearly. The angular momentum for the counterrotating disk in M2 is significantly lower than that for M1. The primary disk in M2 also gains a pronounced bar (not shown), but does not appear to tilt nearly as much as in M1.

The stellar component of the dwarf galaxy also attempts to participate in the counterrotating disk formation, but with much less success. The stellar particles are much more spatially dispersed in both M1 and M2, although there is some flattening discernible in both simulations. In M1, the stellar particles also follow the inclination of the primary disk. The velocity fields of the stars from the dwarf, however, are not at all regular or circularized. In general the angular momenta of the dwarf stars are much lower than those of the counterrotating gas particles, but they are definitely of the same polarity as the gas. So some of the stars do in fact form a counterrotating stellar disk, with more success in M1 than in M2.

The faster pace of M2 apparently leaves less time for the primary disk to tilt in response 
to the incoming material. The presence of the extra dark matter fosters a significantly thicker counterrotating disk than in M1. Surprisingly (see $\S 4.2$ ), the thickness of the primary disk is not affected much by the additional dark matter in M2.

For the third merger simulation (M3), the merger details are the same as for M2, except that the dwarf galaxy is introduced at $t=1.0 \mathrm{Gyr}$ instead of $t=0.5 \mathrm{Gyr}$. This is because the colder primary in M3 requires more time to achieve equilibrium since it is more susceptible to local axisymmetric clumping. The final characteristics of the counterrotating disk are the same as in M2. The main difference between M3 and M2 is in the heating of the primary disk, and this is discussed in the following section.

\section{Discussion}

\subsection{Size, Shape and Orientation of Counterrotating Disks}

Both infall and mergers produce counterrotating disks with sizes which compare well with the observed size of the counterrotating disk in NGC 4138, in terms of mass as well as extent. In some cases, the counterrotating disk is slightly larger in extent than the primary disk, but this is not a big issue because we usually only follow our simulations up to the point where most of the gas falls into the primary disk. The size of the gas disk reduces slowly with time, but we do not wait until it matches that of the primary very closely (to conserve our limited CPU resources, and also because we do not have star formation in our simulations). The mass of the counterrotating disk is usually $\sim 30 \%$ of the mass of the primary disk, since we start with a total secondary mass (in gas for infall and gas+stars for mergers) of $\sim 50 \%$ of the primary disk mass and $\sim 40 \%$ of it is lost in material dispersed over a large volume around the primary galaxy (see below). This is close to the upper limit of the mass of the counterrotating disk derived by JBH.

In the infall simulations, most of the dispersed gas accounts for a very small fraction $(z 5-10 \%)$ of the total gas mass. However, we invariably find one or two large clumps of gas, with masses in the range $\sim 10-30 \%$ of the total gas mass, flung to large distances $(100-200 \mathrm{kpc})$ from the primary disk. The reason for this is that the initial column of infalling gas breaks up into clumps, the last one or two of which are massive enough to survive the tidal field of the primary disk. This also explains why it does not happen in the dwarf merger simulations, although there is a small amount of gas which is spread out over a large volume even in these. The dispersed stars from the dwarf account for the remaining mass which does not form part of the counterrotating disk by the end of the simulations.

We do not see a consistent radial profile for the counterrotating disks formed in our simulations. The radial profiles shown in Fig. 14 are not exponential. The profile for I6 shows a sharp peak at the location of the prograde gas, which has been included. It also shows a higher density for gas at radii above and below the range where the prograde gas is $(\sim 1.5 \pm 0.5 \mathrm{kpc})$. In all the counterrotating disks formed, we see a drop in the density in the nuclear region of the disk. This is true even for I6, so the presence of a small amount of prograde gas does not help. The fact that the size of this "hole" is of the order of the gravitational softening length may be a clue as to its cause. This may also be an indication of the lowest angular momentum achievable by the gas 
as a result of insufficient dissipation in our models (see below). It should be noted, however, that the H I data for NGC 4138 also show a depression in the center (Fig. 3 in JBH).

The thickness of the counterrotating gas disks is also a matter of concern. We find that in the majority of our simulations, the counterrotating disk is somewhat thicker than the primary disk. This is especially so for M2 (and M3). Moreover, the thickness does not reduce appreciably with time on scales of a few dynamical times. One factor that may be causing this is that our gas starts out with a higher velocity dispersion than in TR, and it does not cool as much as it should. There is a cutoff for the collisions for relative velocities of less than $10 \mathrm{~km} / \mathrm{s}$, which is quite low and should allow the gas to cool down. However, if most of the cooling takes place before the gas disk takes shape, the gas will undergo much fewer collisions in the disk state. Alternatively, the thickness may be due to some other limitation of the gas dynamics used.

Except for the inclined infall simulation, in all other cases the counterrotating disk seems to faithfully follow the orientation of the primary disk, even in cases where the primary disk tilts by a considerable amount. This is reassuring but not too surprising, since most of the change in the orientation of the primary seems to occur after the counterrotating disk is mostly formed. The largest disk inclination is seen in infall simulation I5. A calculation of the torques acting on the disk due to the halo and the gas reveals that the rate of change of the disk's angular momentum matches the total torque acting on it. Neither the halo nor the gas can provide enough torque individually to explain the change in the disk's angular momentum. The total torque acting on the disk fluctuates considerably on time-scales as short as a few Myr. We have also calculated the axial ratios and axial vectors of the halo at different times by diagonalizing the normalized inertia tensor,

$$
M_{i j}=\sum m x_{i} x_{j}
$$

where the $x_{i}$ are the components of the unit vector parallel to the radius vector of the particle. There is a slight flattening of the halo along the $z$-axis, and the axial vectors change with time in a manner consistent with the inclination experienced by the disk. The inclination of the disk therefore is intimately linked to the details of the dynamical evolution of the halo and the evolving gas, and different initial conditions are apt to result in very different behavior of the disk.

\subsection{Impact of Accretion on Primary Disk}

If a counterrotating disk forms which resembles the observed disk, then the next question is whether the primary disk suffers a lot of heating as a result of the accretion of the new disk via either infall or a merger. A measure of the heating of the primary disk is its mean thickness as a function of radius. We have plotted the mean half-thickness in Fig. 13 for the important infall and merger simulations. As explained in TR, the primary disk is also heated in the absence of any accretion due to the discreteness of the particle representation and two-body encounters with halo particles. This effect must be taken into account when trying to measure the thickening due to the infall or merger. We have shown a thickness plot for the isolated primary disk evolved up to $t=4.5$ Gyr in addition to the post-accretion plots in Fig. 13. Within $\sim 4 \mathrm{kpc}$, where the thickness measurements are more reliable, the total increase in thickness is not much higher than that due to the numerical heating. When the numerical heating is reduced, as in the case of simulation 
M3, we see a sharp drop in the disk thickening. Since the two effects are not strictly separable, it is hard to make a quantitative statement about the thickening due to accretion only. The best we can say is that the average increase for $R \lesssim 4 \mathrm{kpc}$ is at most of the order of the numerical thickening.

Whereas the results for infall are consistent with the findings of TR, a comparison of Fig. 13 with Fig. 17 in TR reveals that the gas-rich dwarf merger performs much better for NGC 4138 than for the fiducial primary in TR. This is especially remarkable considering that the dwarf galaxy in TR was only $\sim 4 \%$ as massive as the primary galaxy, with its luminous mass being $\sim 10 \%$ of the mass of the primary disk, whereas the corresponding figures for the dwarf in M1 are $\sim 9 \%$ and $\sim 50 \%$. In M2, the total mass of the dwarf is $\sim 18 \%$ of the mass of the primary galaxy. Thus the dwarf galaxies chosen for the NGC 4138 simulations are considerably more massive relative to the primary galaxy than in TR, and the merger time-scales are also much shorter.

The secret to the success of the dwarf mergers in NGC 4138 lies in the nature of its primary disk. The compact and massive primary disk, due to its higher surface density, has a higher vertical velocity dispersion and is therefore initially thicker and much more resistant to vertical heating than the primary used in the simulations by TR. The ratio of scale length to scale height for NGC 4138's disk is only $\sim 5$, compared to $\sim 10$ for the disk used by TR. We chose to model a disk that was radially hot as well because it matched the observed parameters of NGC 4138's current disk well. Of course, the primary disk could have been quite different prior to the formation of the counterrotating disk, and may have been significantly colder radially. This case is represented by M3, where we start with a radially colder disk, and use twice as many collisionless particles to minimize numerical heating. The post-merger disk is now even closer to the observed NGC 4138 disk since it suffers less vertical heating along the way.

\subsection{Comparison with Observations}

The lack of remnants of any tidal features in the vicinity of NGC 4138 may be used as an argument against a dwarf merger origin for the counterrotating disk, but it is not a serious problem. If the merger takes only a few Gyrs to complete, as we have shown, then there has been plenty of time for any tidal features to be absorbed by the primary by now. The extended neutral gas may represent the last of the tidal tails falling back into the primary. The higher mass of the dwarf speeds up the merger considerably. Low-mass satellites would not have been able to spiral in within a Hubble time, especially on a retrograde orbit (Binney \& Tremaine 1987 and Walker, Mihos \& Hernquist 1996, Fig. 13), nor would a single low-mass merger have been able to provide enough mass for the counterrotating disk.

There is nothing in the observations of NGC 4138 to date that precludes gas infall as a means of obtaining a counterrotating disk. The extended neutral gas observed could be the "smoking gun" from the infall process which produced the counterrotating disk. Although there is some uncertainty about the mass of this extended gas, it is not likely to be more than a few percent of the mass of the counterrotating disk, which is consistent with our infall simulations I5 and I6, where the mass of gas around the primary galaxy amounts to a few percent of the mass of the counterrotating disk. The large gas clumps we see at further distances in both of these simulations 
are more problematic, since no counterpart is observed, at least in H I gas, around NGC 4138 (JBH). They owe their existence at least in part to our initial conditions, which allow the tail end of the infalling gas to form clumps dense enough to resist the tidal influence of the primary. Reducing the initial length of the infalling slab of gas by leaving out some of the gas would produce smaller or no leftover clumps of gas.

The presence of A stars along with the absence of $\mathrm{O}$ and $\mathrm{B}$ stars from the primary disk (JBH) suggests that star formation took place less than a Gyr ago in the primary but ceased thereafter. Does the influx of counterrotating gas suppress star formation in the primary? Our simulations indicate that large quantities of counterrotating gas make their way inside the primary disk only at later times, within the last $1-1.5$ Gyr of the simulation. However, keeping in mind that this may be due to a limitation of our models, we cannot rule out the presence of counterrotating gas in the primary disk when the last burst of star formation took place. Although galaxies which harbor counterstreaming gas do not appear to have any ongoing star formation (e.g., Fisher, Illingworth \& Franx 1994), there is no direct proof to date that counterstreaming gas inhibits star formation.

On the other hand, since a small amount of primordial prograde gas in the primary does not cause an appreciable inflow of gas to the center (simulation I6), we are more inclined to believe that collisions between counterstreaming gas clouds trigger star formation rather than inhibiting it. Incoming counterrotating gas would generally have the larger specific angular momentum, as demonstrated in Fig. 15. The direction of the orbits acquired by stars resulting from the interaction between corotating and counterrotating gas would depend upon the relative masses of the two gas components. A more massive prograde component should produce significantly more stars with prograde orbits. Small amounts of prograde gas would produce stars with mostly retrograde orbits. In either case, some gas will flow inwards.

Under the above hypothesis, the A stars seen in the primary disk are an indication that counterrotating gas was present throughout the primary disk less than a Gyr ago. The star formation would have proceeded inwards as more and more counterrotating gas found its way there, ending earlier at radii where prograde gas was exhausted faster. The annulus of counterrotating $\mathrm{G}$ and $\mathrm{K}$ giants around the $\mathrm{H} \alpha$ ring supports these ideas. The radial distribution of corotating $\mathrm{G}$ and $\mathrm{K}$ giants reflects the primordial radial distribution of prograde gas (decreasing smoothly from the center outwards).

If the counterrotating gas owes its origin to a merger instead of infall, at least some of the counterrotating $\mathrm{G}$ and $\mathrm{K}$ giants could have been inherited from the dwarf galaxy. In our dwarf merger simulations, we found that even though the stars from the dwarf have net counterrotation, they form an oblate structure which resembles an extended (radius $\gtrsim 3$ times radius of primary) and rather thick (scale height $\sim 2-3 \mathrm{kpc}$ ) disk, rather than the compact and flat disk formed by the gas. This distinction is clearer in M1 where the gas disk is flatter. Observationally, such an extended counterrotating stellar population may be hard to separate from the halo stars and hence missed altogether.

Figs. 16 and 17 show the final rotation curves for an infall (I5) and dwarf merger (M1) simulation respectively. The counterrotating gas is colder than the primary stars. The rotation curve of the counterrotating gas is slightly higher than that of the primary, and its velocity dispersions are systematically lower than those of the primary stars. This is qualitatively in 
agreement with the observed rotation curves, but our velocity dispersion values for the primary disk stars do not agree well with the observed values reported by JBH. Our values are significantly lower, in some cases by as much as a factor of 2 . The velocity dispersion values for the counterrotating gas agree with the observations much better. Since our gas never gets converted into stars, we do not have values for the velocity dispersions for counterrotating stars.

\subsection{Comparison with Previous Work}

The heating experienced by our primary disk after mergers with dwarfs that have half its own mass (not counting the dark matter) is modest compared with that due to a dissipationless merger between a large disk galaxy like the Milky Way, and a dense dwarf galaxy with one-tenth the mass of the primary disk (Walker, Mihos \& Hernquist 1996). The latter causes the disk to thicken by $\sim 60 \%$ at the solar circle, which is not surprising considering that the merger is dissipationless, the primary is cold and thin, and a good portion of the satellite's mass $(\sim 45 \%)$ survives intact and sinks to the center of the primary. It is also a prograde merger which completes rapidly, in less than 3 Gyr. This is due to the interaction of the satellite with the disk particles, caused by the small initial distance of the satellite from the disk (6 disk scale lengths). There is not enough time for the primary to completely tidally strip the satellite and thereby transfer orbital energy to the tidal remnants. The halo also does not have the opportunity to absorb some of this energy. A large initial orbital momentum merger (in which the satellite starts out at several disk radii) of a $10 \%$ mass satellite, by contrast, is much less damaging to the primary (Huang \& Carlberg 1996). This is more in agreement with our simulations, in which the infalling gas as well as the dwarf galaxy are initially placed at a large average distance $(\sim 20$ disk radii $)$ from the primary disk. The relatively low density of the satellite in each of our mergers (central density of primary is $\sim 40$ times the central density of the dwarf) also contributes to more efficient stripping of the satellite.

The higher initial angular momentum and lower density satellites are only part of the story, however. As mentioned in $\S 4.2$, the nature of the primary in NGC 4138 has a lot to do with the amount of heating experienced. The fragility of cold and thin galactic disks has been demonstrated time and again in the context of minor mergers by numerical (TR; Walker, Mihos \& Hernquist 1996; Quinn, Hernquist \& Fullagar 1993; Quinn \& Goodman 1986) and analytical (Toth \& Ostriker 1992; Quinn \& Goodman 1986) studies, but the response of early-type disk galaxies like NGC 4138 to minor mergers has not received much attention. The resistance of these disks to minor mergers appears to be significantly higher. The production of counterrotating disks and bulges via retrograde minor mergers is much more likely for S0s and Sas than for later type spirals. The counterrotating bulge recently reported in the Sb galaxy NGC 7331 (Prada et al. 1996) is most likely a result of very low angular momentum gas infall rather than a minor merger.

The origin of the gas for continuous infall is an open question, and for this reason the dwarf merger process is potentially a more frequent producer of counterrotating disks. The formation of dwarf galaxies in tidal tails of interactions between larger galaxies has numerical Barnes \& Hernquist 1992b; Elmegreen, Kaufmann \& Thomasson 1993; Hibbard \& Mihos 1995) as well as observational (Hibbard et al. 1994; Hunsberger, Charlton \& Zaritsky 1996) support. It provides

a possible mechanism for obtaining gas-rich dwarf galaxies which have less dark matter than the 
norm. The tidal features observed (Hibbard et al. 1994) in NGC 7252 (the "Atoms for Peace" galaxy), for instance, contain clumps with properties typical of dwarf irregular galaxies in terms of their high atomic gas content, luminosity, velocity width and $M / L$ ratios $(\sim 1.5-7.5$ for $\left.50 \leq H_{\circ} \leq 75\right)$. These tidally formed dwarfs, however, are substantially less massive than the dwarfs used in simulations M1-M3.

The S0 galaxy NGC 7332 (Fisher, Illingworth \& Franx 1994) shows extended counterstreaming [O III] gas in which the counterrotating gas has higher velocity than the prograde gas and stars. This is cited as support for recent accretion and agrees with the behavior seen in our infall simulation with prograde gas (I6), where we find that the counterrotating gas has higher angular momentum than the corotating gas. We do not, however, see any clear gas motion towards the center as a result of the interaction of the counterstreaming components. An analytical study of two counterrotating gas disks lying one over the other predicts that the counterrotating gas should drag the corotating gas inward with equal masses of corotating and counterrotating gas accreting rapidly (Lovelace \& Chou 1996). Although we see enhanced accretion for infall with prograde gas

(I6), the amount of prograde gas in our test is small and cannot be expected to dramatically affect the accretion and inflow rate of the counterrotating gas. We do however see some evidence of the pile-up of gas into rings at one or more radial distances predicted by Lovelace, Jore \& Haynes (1996) as a consequence of a two-stream instability in counterrotating systems. This is evident in Fig. 14, especially for M2 and I6, where local peaks and troughs dominate the radial gas profile.

The evolution of the tidal material seen in our simulations is in general agreement with the behavior described by Hibbard (1995) as a generic result of mergers. For the lowest orbital angular momentum case (I3), we see the tidal material falling back early in shells, and as the orbital angular momentum increases, so does the fallback time as the tidal material forms larger and larger loops. We also confirm that all the tidal material remains bound and eventually will fall back into the primary galaxy. Nested, counterrotating, stable bars within primary bars have been observed in simulations of retrograde gas-rich dwarf mergers where the primary is an early-type barred galaxy (Friedli 1996), but we do not see this happening in our simulations. One reason for this is that there is not much counterrotating gas collecting in the center of the primary disk.

\section{Conclusions}

Based on numerical simulations carried out using a fiducial spiral galaxy and infall/merger model, TR predicted that counterrotating disks should be a rare occurence because they require special conditions which must persist over several Gyrs. This was partly due to the fact that dwarf merger simulations were not able to produce counterrotating disks with the required properties within the required time frame. Our experiments with NGC 4138 lead us to be more optimistic about the chances of forming counterrotating disks with dwarf mergers. The combined effects of a massive, compact primary and a smaller dynamical time (due to the smaller size of the primary) gives a dwarf merger a much better chance at success. For a dwarf merger model based on the observationally determined parameters of the galaxy, we have found a configuration which produces a counterrotating disk closely matching the one observed, although it does require the dwarf galaxy to be predominantly gaseous with little to moderate amounts of dark matter. 
The results of minor mergers involving large primary galaxies with cold, thin disks cannot be extended to all types of spirals. Early type spirals, with hot, massive and compact disks of the type that NGC 4138 has, appear to be more resistant and less fragile. The initial orbital angular momentum of the satellite plays a role in determining the damage done to the primary disk as well in numerical simulations, and it must be taken into account when interpreting the results.

Continuous infall also succeeds in producing a counterrotating disk which matches the observed one in terms of mass and size. The time frame for infall is somewhat longer than for dwarf mergers. Whereas a dwarf merger yields a counterotating disk in $3-4$ Gyr, the infall process accomplishes this in $\sim 5$ Gyr.

A common characteristic of the counterrotating gas disk resulting from both infall and mergers is that it does not have an exponential profile like the primary disk. This is not a problem for NGC 4138, since there is no evidence of its counterrotating disk having an exponential profile, but counterrotating disks in some other galaxies do have exponential profiles (e.g., NGC 4550, NGC 3593).

The nuclear star formation ring may owe its origin to an interaction between prograde and retrograde gas or to a pile up of gas at a resonance. The counterstreaming gas hypothesis is viable for both infall and mergers. The inferred star formation histories of the primary and counterrotating disks do not pose serious problems for this hypothesis currently, but knowledge of the colors and ages of the counterrotating stars is necessary to resolve this issue.

As pointed out by JBH, we have only tested a restricted set of infall and merger possibilities so far. This is partly because these processes are not well constrained observationally (especially infall), but also because of our CPU resource limitations. There is a need to explore alternative infall and merger models before a reliable prediction can be made regarding the overall ease of obtaining massive CR disks in all types of spiral galaxies.

A.R.T. thanks Joshua Barnes for providing the $\mathrm{C}$ version of the basic tree code, and Richard Pogge and Glenn Tiede for useful discussions. We thank Richard Pogge for comments on the manuscript. Support for this work was provided by a NYI award to B.S.R. (NSF grant AST-9357396), NASA grant NAG 5-2864, and an Ohio Supercomputer Center Research Grant (PAS825) for computer time on the Cray Y-MP. We wish to thank the O.S.C. staff for their assistance. Finally, we thank the referee, Chris Mihos, for providing a thorough and insightful referee report.

\section{REFERENCES}

Balcells, M., \& Quinn, P.J. 1990, ApJ, 361, 381.

Barnes, J.E. 1992, ApJ, 393, 484.

Barnes, J.E., \& Hernquist, L.E. 1991, ApJ, 370, L65.

Barnes, J.E., \& Hernquist, L.E. 1992a, ARA\&A, 30, 705. 
Barnes, J.E., \& Hernquist, L.E. 1992b, Nature, 360, 715.

Barnes, J.E., \& Hut, P. 1986, Nature, 324, 446.

Bertola, F., Cinzano, P., Corsini, E.M., \& Pizzella, A. 1996, ApJ, 458, L67.

Binney, J., \& Tremaine, S. 1987, Galactic Dynamics (Princeton University Press), $§ 7.1$ and eq. 7-27.

Brahic, A. 1977, A\&A, 54, 895.

Braun, R., Walterbos, R.A.M., \& Kennicutt, R.C. Jr 1992, Nature, 360, 442.

Ciri, R., Bettoni, D., \& Galletta, G. 1995, Nature, 375, 661.

de Vaucouleurs, G., de Vaucouleurs, A., Corwin, H.G., Buta, R.J., Paturel, G., \& Fouque, P. 1991, Third Reference Catalogue of Bright Galaxies (Springer, New York). Galletta, G. 1995, Nature, 375, 661.

Elmegreen, B., Kaufmann, M. \& Thomasson, M. 1993, ApJ, 412, 90.

Fisher, D., Illingworth, G. \& Franx, M. 1994, AJ, 107, 160.

Friedli, D. 1996, A\&A, in press.

Galletta, G. 1987, ApJ, 318, 531.

Hernquist, L.E., \& Barnes, J.E. 1991, Nature, 354, 210.

Hibbard, J.E. 1995, Ph.D. Thesis, Columbia University, Chs. IV and VI.

Hibbard, J.E., Guhathakurta, P., van Gorkom, J.H., \& Schweizer, F. 1994, AJ, 107, 67.

Hibbard, J.E., \& Mihos, J.C. 1995, AJ, 110, 140.

Huang, S. \& Carlberg, R.G. 1996, ApJ, submitted.

Hunsberger, S.D., Charlton, J.C., \& Zaritsky, D. 1996, ApJ, submitted.

Jore, K.P., Broeils, A.H., \& Haynes, M.P. 1996, ApJ, submitted. [JBH]

Kuijken, K., Fisher, D., \& Merrifield, M.R. 1996, MNRAS, in press.

Lovelace, R.V.E., \& Chou, T. 1996, ApJ, submitted.

Lovelace, R.V.E., Jore, K.P., \& Haynes, M.P. 1996, ApJ, submitted.

Merrifield, M.R., \& Kuijken, K. 1994, ApJ, 432, 575.

Negroponte, J., \& White, S.D.M. 1983, MNRAS, 208, 1009.

Pogge, R.W. \& Eskridge, P.B. 1987, AJ, 93, 291.

Pogge, R.W. \& Eskridge, P.B. 1993, AJ, 106, 1405. 
Prada, F., Gutierrez, C.M., Peletier, R.F., \& McKeith C.D. 1996, MNRAS, submitted.

Quinn, P.J. \& Goodman, J. 1986, ApJ, 309, 472.

Quinn, P.J., Hernquist, L.E., \& Fullagar, D.P. 1993, ApJ, 403, 74.

Roberts, M.S. \& Haynes, M.P. 1994, ARA\&A, 32, 115.

Rubin, V.C., Graham, J.A., \& Kenney, J.D.P. 1992, ApJ, 394, L9.

Sage, L.J. \& Galletta, G. 1994, AJ, 108, 1633.

Sandage, A.R., and Tammann, G.A. 1981, A Revised Shapley-Ames Catalog of Bright Galaxies, (Washington, Carnegie Institution of Washington).

Schwarz, M.P. 1981, ApJ, 247, 77.

Toth, G. \& Ostriker, J.P. 1992, ApJ, 389, 5.

Thakar, A.R., \& Ryden, B.S. 1996, ApJ, 461, 55. [TR]

Thakar, A.R. 1996, BAAS, 28, 959.

Walker, I.R., Mihos, J.C., \& Hernquist, L.E. 1996, ApJ, 460, 121. 
Fig. 1.- The initial rotation curves for the primary disk and halo in our numerical model compared with the corresponding rotation curves derived from observations of NGC 4138 (JBH).

Fig. 2.- Gas infall with large initial angular momentum for the gas (I1). The top half shows the "top" view and the bottom half shows the "side" view. The primary disk is shown only in the first panel for each view for clarity. The galactic halo is not shown. The mass of the gas is $50 \%$ of the mass of the disk. The square panels are $80 \mathrm{kpc}$ on each side, except for the last panel, which is 40 kpc on each side. Time is in Gyr.

Fig. 3.- Top (upper half) and side (lower half) view of gas infall with just just enough initial angular momentum to still yield a counterrotating disk (I2). Panel widths are $80 \mathrm{kpc}$ except for the last panel in each view, which is $40 \mathrm{kpc}$ wide.

Fig. 4.- Top (upper half) and side (lower half) view of gas infall with very low initial angular momentum (I3). Panel widths are $80 \mathrm{kpc}$ except for the last panel in each view, which is $40 \mathrm{kpc}$ wide.

Fig. 5.- Velocity fields of the disk (bottom) and counterrotating gas (top) compared for I3 (left) vs. I1 (right). Only a fraction of the particles are sampled, and sampling is proportional to radius.

Fig. 6. - The mean specific angular momentum of the disk and counterrotating gas compared for I3 (open squares) and I1 (filled squares). In our sign convention, $J_{z}>0$ for prograde orbits and $J_{z}<0$ for retrograde orbits.

Fig. 7.- Top (far left) and side (left) views of the gas, and top (right) and side (far right) views of the primary disk for gas infall with an orbital inclination of $\sim 30^{\circ}$ (I4). Panel widths are in kpc.

Fig. 8. - Top (left) and side (center) views of the gas, and side views of the primary disk (right), for continuous gas infall with a smaller search radius for sticky particle neighbors (I5). The primary disk is shown for reference also in the first panel of each of the gas views. For the gas, the square panels are $80 \mathrm{kpc}$ wide, with the last one in each view being $20 \mathrm{kpc}$ wide. For the primary disk, all panels are $20 \mathrm{kpc}$ wide.

Fig. 9.- Top (upper half) and side (lower half) view of continuous gas infall with a prograde ring of gas in the primary disk initially at $\sim 1.2 \mathrm{kpc}$ from the center (I6). The prograde gas is also shown. The last two panels show magnified views of the counterrotating disk. The square panels are $80 \mathrm{kpc}$ wide, except for the last one, which is $20 \mathrm{kpc}$ wide.

Fig. 10. - Top (upper half) and side (lower half) views of a gas-rich dwarf merger with very little dark matter in the dwarf (M1). The primary disk is shown as light grey, and both the stars and the gas in the dwarf are shown as dark particles. The dark halos are not shown. The square panels are $80 \mathrm{kpc}$ wide, except for the last panel, which is $40 \mathrm{kpc}$ wide.

Fig. 11. - Top (upper half) and side (lower half) views of a gas-rich dwarf merger (M2) with 4 times the amount of dark matter as in M1. 
Fig. 12.- A comparison of the two dwarf mergers M1 and M2, showing three species: the primary disk (left), the gas in the dwarf (center) and the stars in the dwarf (right). The angular momentum comparison plots are in the middle (open squares for M1, filled squares for M2), with the velocity fields and the side views for M1 above the A.M. plots, and the velocity fields and side views for M2 below them.

Fig. 13. - A comparison of the thickness of the disk after continuous infall and dwarf mergers. The solid line shows the initial disk thickness at $t=0$. The dotted line is the disk thickness at $t=4.5$ Gyr for an isolated disk evolved without any infall or merger. Thicknesses for $R \gtrsim 4 \mathrm{kpc}$ are not reliable due to small numbers of particles at larger disk radii.

Fig. 14.- A comparison of the radial mass distribution of the counterrotating disk for infall and dwarf mergers. The $y$-axis shows the number density $N$ of gas particles.

Fig. 15. - A comparison of the mean specific angular momentum of the counterrotating gas (open squares) with that of the corotating (prograde) gas for I6.

Fig. 16. - The final rotation curves and velocity dispersion plots for the primary disk (filled points) and the counterrotating gas (open points) after gas infall (I5). The initial disk rotation curve is drawn in for comparison. The velocity dispersion values are somewhat smaller than the observed values. The disk inclination is taken into account in calculating the rotation speed.

Fig. 17. - The final rotation curves and velocity dispersion plots for the primary disk (filled points) and the counterrotating gas (open points) for the dwarf merger M1. The initial disk rotation curve is drawn in for comparison. Both the rotation curves and the velocity dispersion values are higher than those for the infall simulation, although part of the difference may be due to the error in the inclination correction. The dispersion values are closer to the observed values in this case. 
Table 1: PARAMETERS FOR NGC 4138 MODEL.

\begin{tabular}{cccccccc}
\hline \hline & \multicolumn{2}{c}{ Disk } & & \multicolumn{3}{c}{ Halo } \\
\cline { 1 - 1 } \cline { 6 - 8 } Mass & Radius & Scale Len. & Scale Ht. & & Mass & Radius & Core Rad. \\
$M_{d}$ & $R_{D}$ & $R_{d}$ & $h_{d}$ & & $M_{h}$ & $R_{h}$ & $R_{c}$ \\
\hline $2.0 \times 10^{10}$ & 6.0 & 1.25 & 0.2 & & $1.5 \times 10^{11}$ & 20 & 5 \\
\hline
\end{tabular}

Note. - Masses are in $M_{\odot}$, lengths in kpc.

Table 2: PARAMETERS FOR GAS INFALL SIMULATIONS.

\begin{tabular}{lcccccc}
\hline \hline Model & $M_{g}{ }^{\mathrm{a}}$ & $V_{0}{ }^{\mathrm{b}}$ & $X_{0}{ }^{\mathrm{c}}$ & $J_{z}^{\mathrm{d}}$ & $r_{\mathrm{nn}}{ }^{\mathrm{e}}$ & $Z_{0}{ }^{\mathrm{f}}$ \\
\hline I1 & $1.0 \times 10^{10}$ & 0.3 & 25 & 0.45 & 3.0 & 0 \\
$\mathrm{I} 2$ & $1.0 \times 10^{10}$ & 0.3 & 10 & 0.18 & 3.0 & 0 \\
$\mathrm{I} 3$ & $1.0 \times 10^{10}$ & 0.2 & 10 & 0.12 & 3.0 & 0 \\
$\mathrm{I} 4$ & $1.0 \times 10^{10}$ & 0.3 & 20 & 0.36 & 3.0 & 15 \\
$\mathrm{I} 5$ & $1.0 \times 10^{10}$ & 0.3 & 25 & 0.45 & 1.0 & 0 \\
$\mathrm{I} 6$ & $1.1 \times 10^{10 \dagger}$ & 0.3 & 25 & 0.45 & 1.0 & 0 \\
\hline
\end{tabular}

atotal gas mass, in $M_{\odot}$

${ }^{\mathrm{b}}$ the initial velocity of the gas in the negative $y$ direction, specified in units of the centripetal velocity $\sqrt{G\left(M_{h}+M_{d}\right) / X_{0}}$, where $M_{h}+M_{d}$ is the total mass of the primary (halo+disk)

${ }^{c}$ the initial distance of the gas column from the center of the primary disk, in kpc

${ }^{d}$ the magnitude of the angular momentum (quantified crudely by taking the product $m_{g} \times V_{0} \times R_{0}$, where $m_{g}$ is the total mass of the infalling gas in our simulation units)

${ }^{e}$ the search radius for nearest neighbors used by the sticky particle code, in $\mathrm{kpc}$

${ }^{\mathrm{f}}$ the initial height of the gas column above the $x-y$ plane, in kpc

${ }^{\dagger}$ I6 has additional prograde gas in the primary disk with one-tenth the mass of the infalling gas. 
Table 3: DWARF GALAXY PARAMETERS FOR GAS-RICH DWARF MERGERS.

\begin{tabular}{lccccccc}
\hline \hline Model & $M_{g}{ }^{\mathrm{a}}$ & $M_{*}{ }^{\mathrm{b}}$ & $M_{D}{ }^{\mathrm{c}}$ & $M_{g} / M_{T}{ }^{\mathrm{d}}$ & $R_{*}{ }^{\mathrm{e}}$ & $R_{g}{ }^{\mathrm{f}}$ & $R_{h^{\mathrm{g}}}$ \\
\hline M1 & $8 \times 10^{9}$ & $2 \times 10^{9}$ & $5 \times 10^{9}$ & 0.53 & 4.0 & 4.0 & 4.0 \\
M2 & $8 \times 10^{9}$ & $2 \times 10^{9}$ & $2 \times 10^{10}$ & 0.27 & 4.0 & 4.0 & 8.0 \\
M3 & $8 \times 10^{9}$ & $2 \times 10^{9}$ & $2 \times 10^{10}$ & 0.27 & 4.0 & 4.0 & 8.0 \\
\hline
\end{tabular}

a mass of gas, in $M_{\odot}$

${ }^{\mathrm{b}}$ mass of stars, in $M_{\odot}$

${ }^{\mathrm{c}}$ mass of dark matter, in $M_{\odot}$

${ }^{\mathrm{d}}$ ratio of gas mass to total mass

${ }^{\text {e}}$ radius of stellar sphere, in $\mathrm{kpc}$

${ }^{\mathrm{f}}$ radius of gas sphere, in $\mathrm{kpc}$

${ }^{\mathrm{g}}$ radius of halo, in $\mathrm{kpc}$

${ }^{\mathrm{h}} \mathrm{M} 3$ uses twice the number of particles as M2 


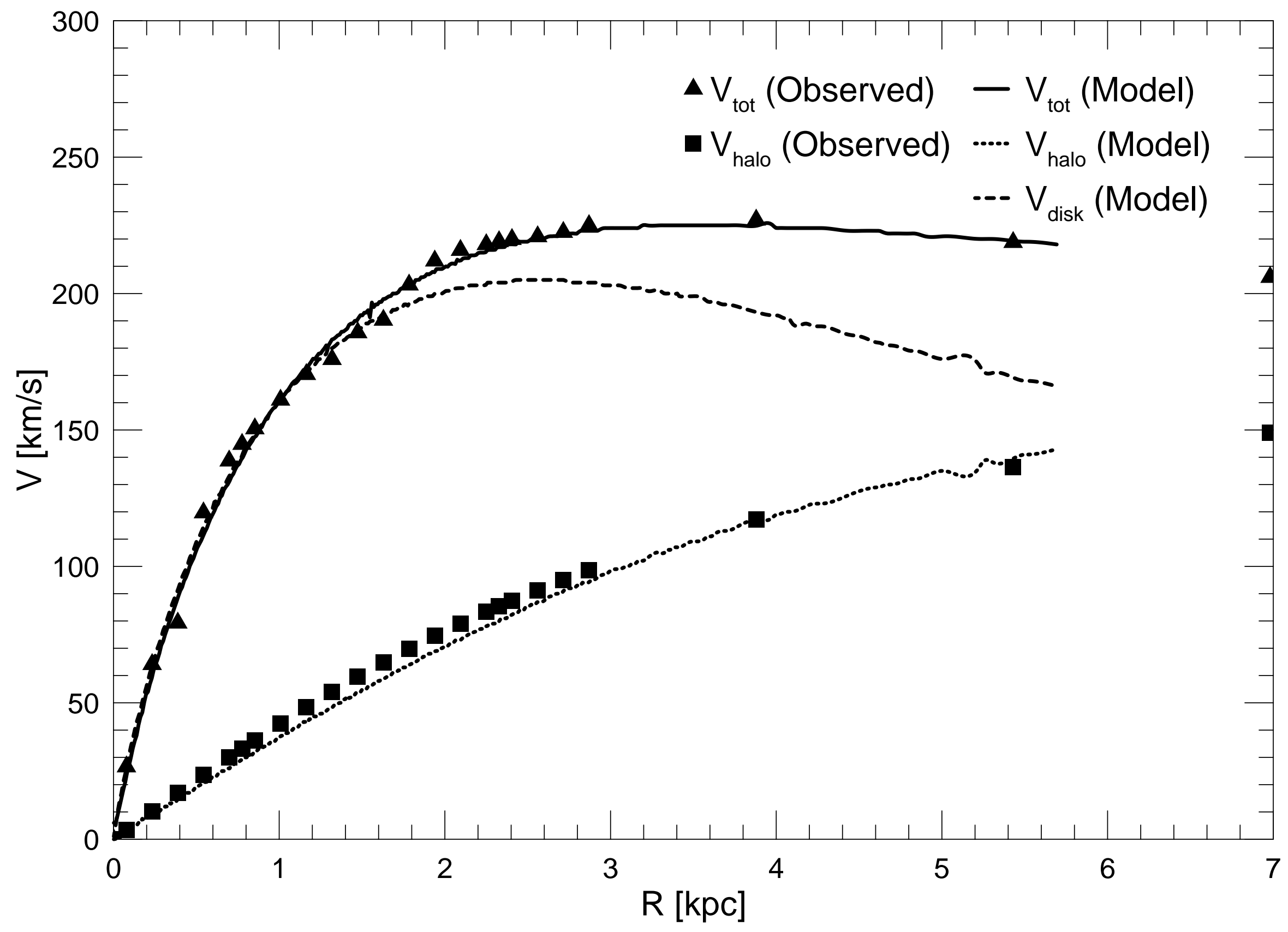


This figure "fig2.gif" is available in "gif" format from: http://arxiv.org/ps/astro-ph/9611042v1 
This figure "fig3.gif" is available in "gif" format from: http://arxiv.org/ps/astro-ph/9611042v1 
This figure "fig4.gif" is available in "gif" format from: http://arxiv.org/ps/astro-ph/9611042v1 
This figure "fig5.gif" is available in "gif" format from: http://arxiv.org/ps/astro-ph/9611042v1 


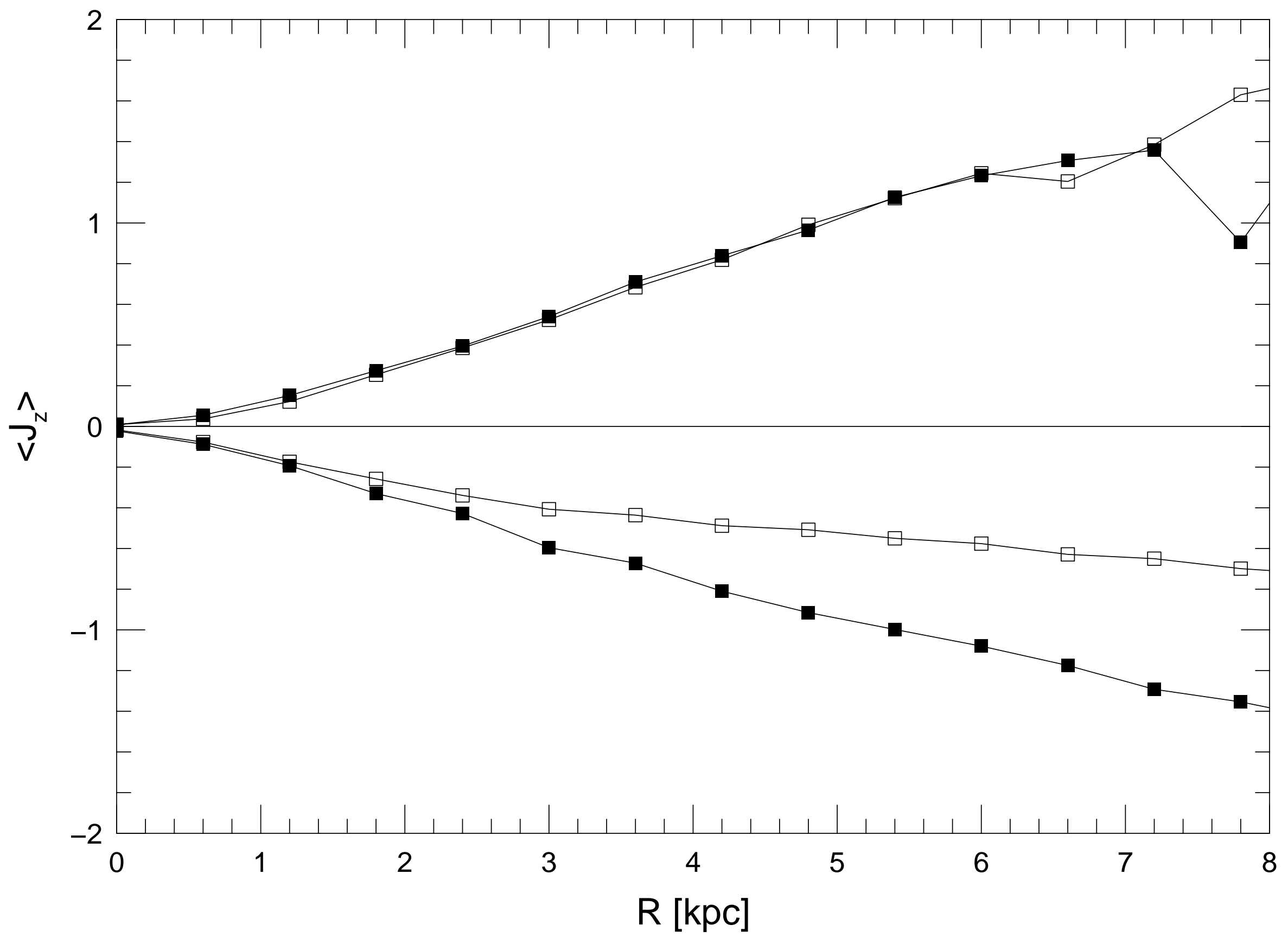


This figure "fig7.gif" is available in "gif" format from: http://arxiv.org/ps/astro-ph/9611042v1 
This figure "fig8.gif" is available in "gif" format from: http://arxiv.org/ps/astro-ph/9611042v1 
This figure "fig9.gif" is available in "gif" format from: http://arxiv.org/ps/astro-ph/9611042v1 
This figure "fig10.gif" is available in "gif" format from: http://arxiv.org/ps/astro-ph/9611042v1 
This figure "fig11.gif" is available in "gif" format from: http://arxiv.org/ps/astro-ph/9611042v1 


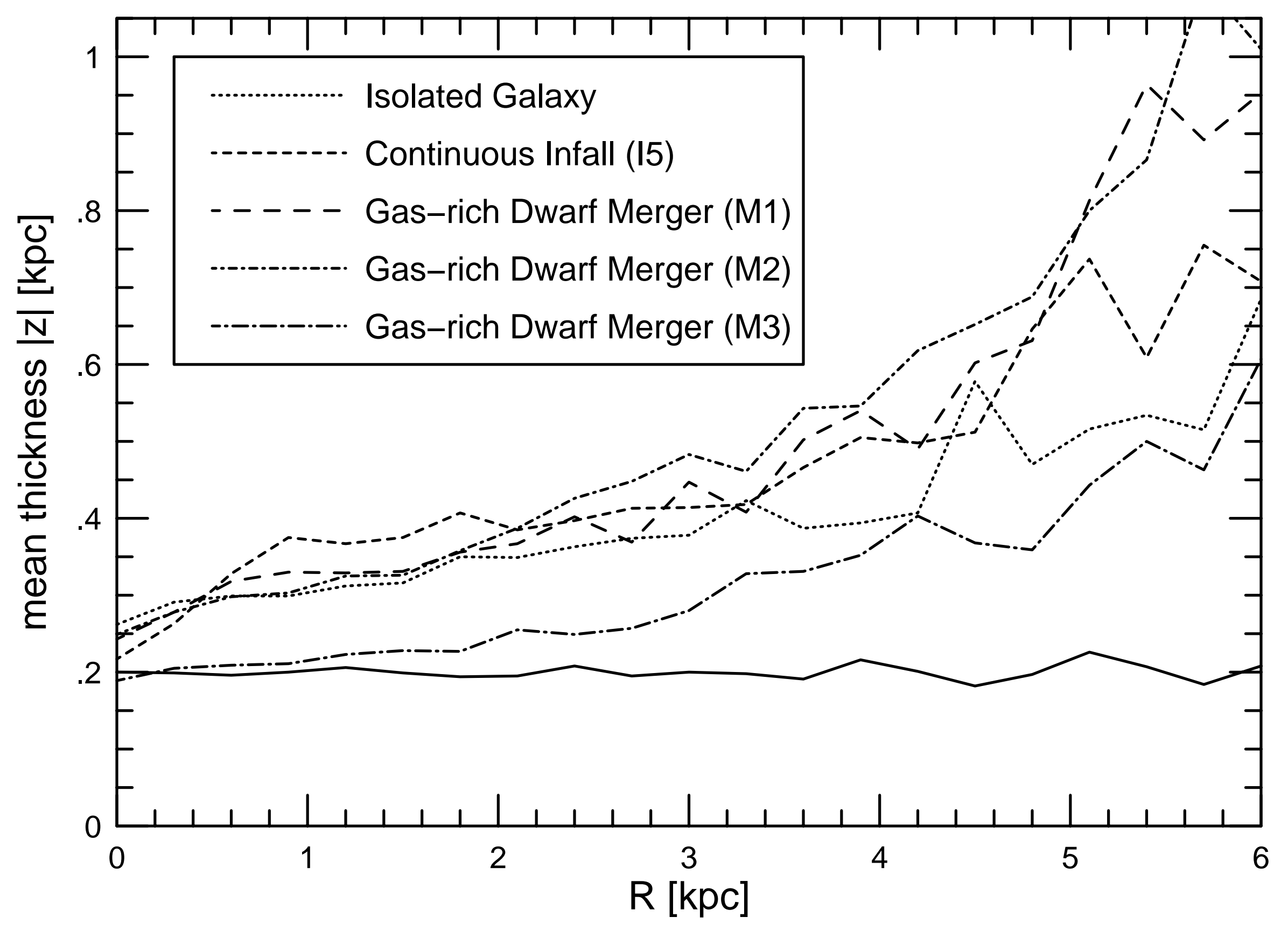




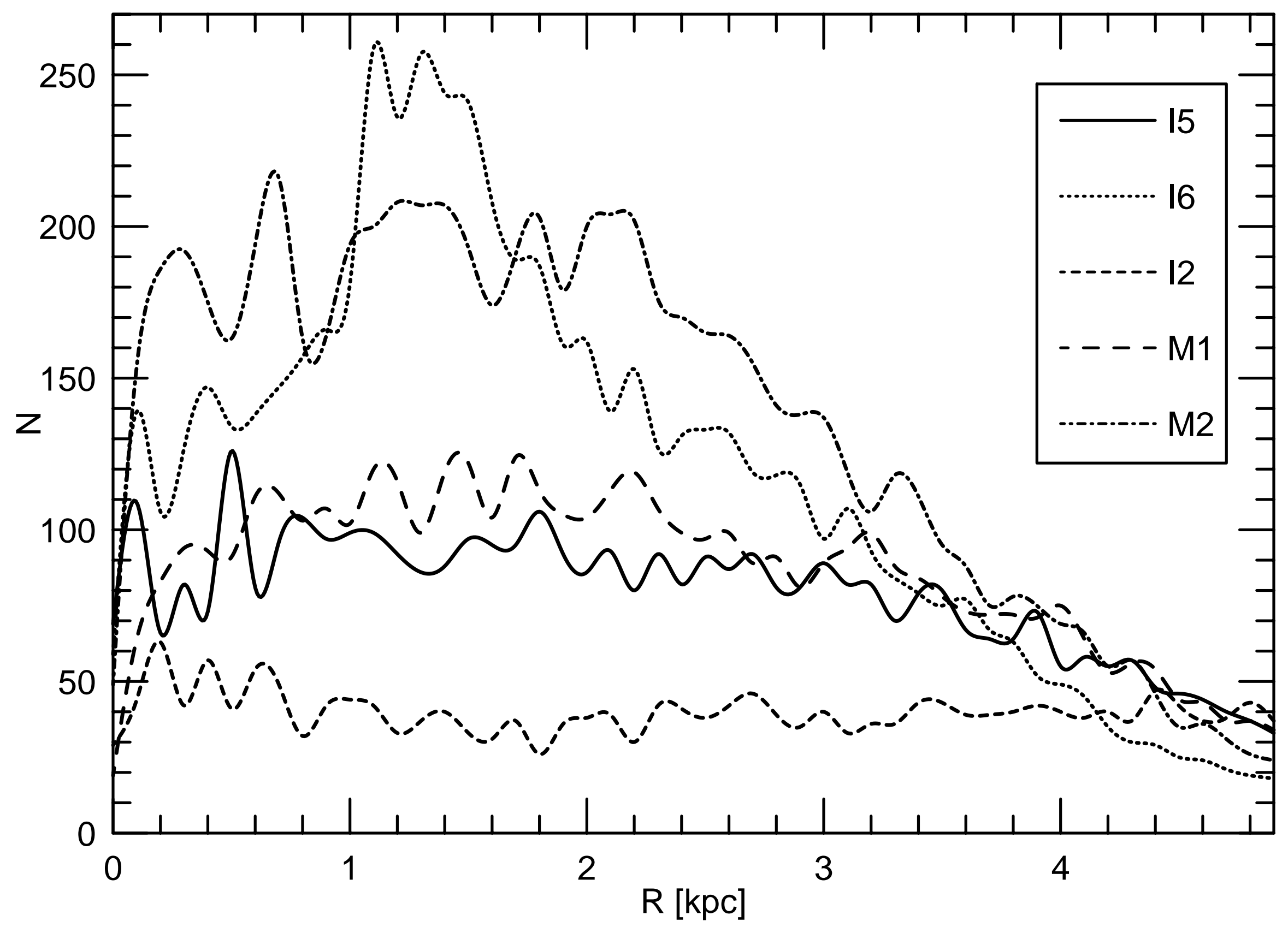




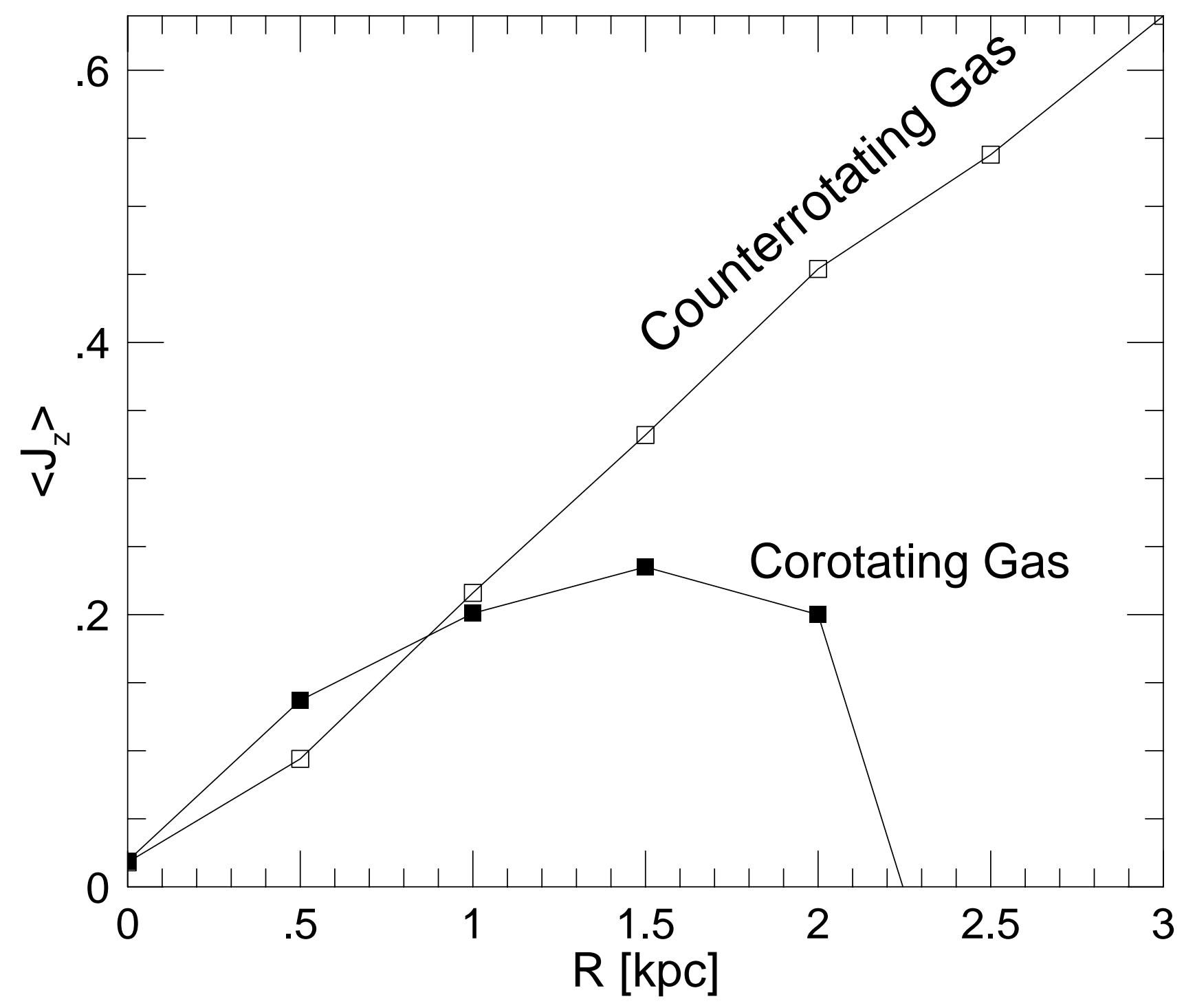




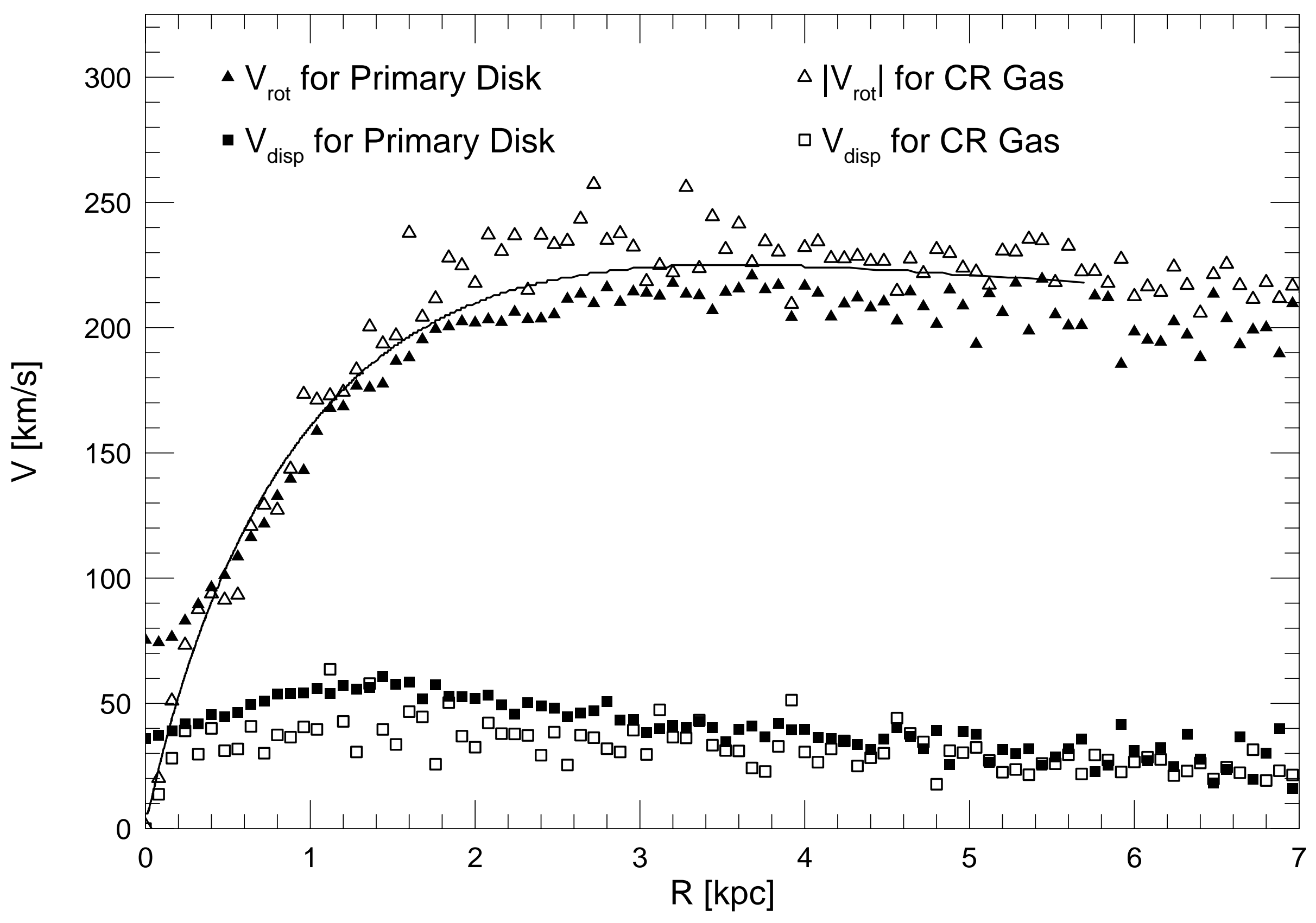




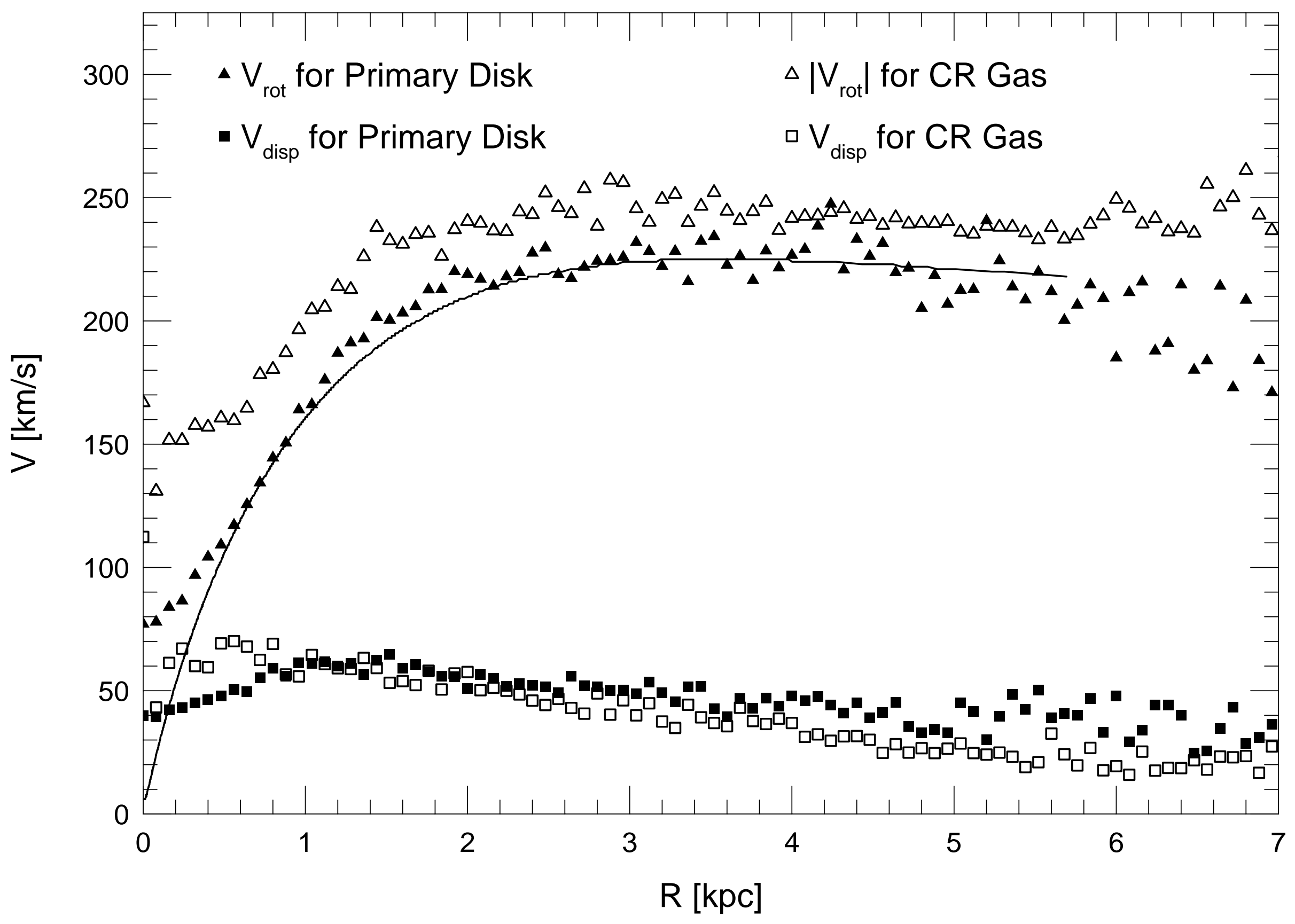

